\title{
Evolution of magnetic resonance imaging features in cerebral parenchyma from prolonged focal status epilepticus: a case study
}

\author{
Sung Chul Lim ${ }^{1}$, Jung Hee Cho ${ }^{1}$, Young-Min Shon ${ }^{2}$ \\ ${ }^{1}$ Department of Neurology, St. Vincent's Hospital, College of Medicine, The Catholic University of Korea, Seoul, Korea \\ ${ }^{2}$ Department of Neurology, Samsung Medical Center, Sungkyunkwan University School of Medicine, Seoul, Korea
}

\begin{abstract}
It has rarely been documented that permanent alteration of cerebral structures occurs by focal status epilepticus (FSE). We report the case of a 16-year-old boy with FSE in whom serial T1-weighted magnetic resonance volumetry and conventional magnetic resonance imaging were useful for investigating an evolving pattern of morphological changes during and after the FSE, including cortical laminar necrosis (CLN), increased T2 signal intensities, and marked regional atrophy on the corresponding areas. Despite cessation of FSE after adequate medication (combination therapy including clobazam of $1 \mathrm{mg} / \mathrm{kg} /$ day), further significant cerebral atrophy was detected at the limited regions where discrete CLN had occurred during the FSE.
\end{abstract}

Keywords: Status epilepticus, Brain atrophy, Hippocampal atrophy, Ictal hyperperfusion

\section{Introduction}

Transient signal abnormalities of magnetic resonance imaging (MRI) have been attributed to functional changes due to seizure activity. Periictal cerebral imaging can show reversible abnormalities, and postictal diffusion-weighted MRI (DWI) can be used to delineate epileptic areas [1].

Likewise, in patients with status epilepticus (SE), transient and permanent changes of micro- or macroscopic brain structures by persistent ictal discharges have been demonstrated $[2,3]$. Moreover, hemodynamic and ultrastructural changes in areas remote from the site of primary epileptogenic activity have been reported in generalized and focal SE such as the hippocampal formation, thalamus, and cerebellum $[4,5]$. Comparative or quantitative morphological changes caused by prolonged SE have not been well investigated, es- pecially those resulting from FSE.

We report an interesting case in which distinct morphological evolution was observed by conventional MRI and magnetic resonance (MR) volumetric measurement in the course of exceptionally prolonged and uncontrolled FSE.

\section{Case Report}

A 16-year-old male was admitted to the pediatric department of our hospital for two recent episodes of bilateral tonic clonic seizure (BTCS) in January 2015. He had undergone two bone marrow transplantations for acute myelogenous leukemia (M3) and had been maintained on immunosuppressant therapy with tacrolimus and mycofenolate mofetil since August 2014. In December 2014, he had experienced a few episodes of eyeball deviation to the left side with or without brief loss of

Received: September 27, 2021 Revised: October 25, $2021 \quad$ Accepted: November 1, 2021

Correspondence: Young-Min Shon

Department of Neurology, Samsung Medical Center, Sungkyunkwan University School of Medicine, 81 Irwon-ro, Gangnam-gu, Seoul 06351, Korea

E-mail: youngmin.shon@samsung.com

ORCID: https://orcid.org/0000-0001-5171-1695

Copyright (C) 2022 by The Korean Encephalitis and Neuroinflammation Society

This is an open access article distributed under the terms of the Creative Commons Attribution Non-Commercial License (http://creativecommons.org/licenses/by-nc/4.0/) which permits unrestricted non-commercial use, distribution, and reproduction in any medium, provided the original work is properly cited. 
consciousness and without secondary BTCS. At the first admission (department of pediatrics of our hospital), his blood pressure and heart rate were normal and mental status was intact. Also, there was no definite focal change in his first MRI scan (M1) except for minimal, diffuse cortical atrophy and ventricular dilation bilaterally (Figure 1B). Electroencephalogram (EEG) showed a few spikes with regional slowing in both occipital areas. Although the typical findings in posterior brain areas of reversible posterior leukoencephalopathy syn- drome (RPLS) were not seen on MRI, neither the normal serum level of tacrolimus $(5.1 \mu \mathrm{g} / \mathrm{L})$ nor the dose of tacrolimus was reduced in anticipation of tacrolimus-induced RPLS. After antiepileptic medication with carbamazepine (200 mg/ day), no additional motor seizures occurred, and the patients were discharged without any sequelae. However, at 3 months after discharge, he complained of a newly developed visual symptom described as near-constant, unformed, fragmented flashes of multicolored lights in the left upper visual field. The

Figure 1 Conspicously changing pattern of electroencephalogram, MRI, and SPECT findings performed before, during, and after FSE

A

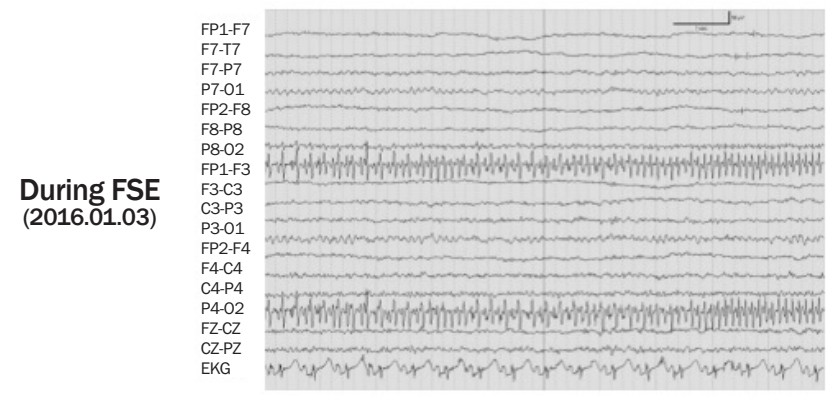

B

Baseline (2015.01.19) M1
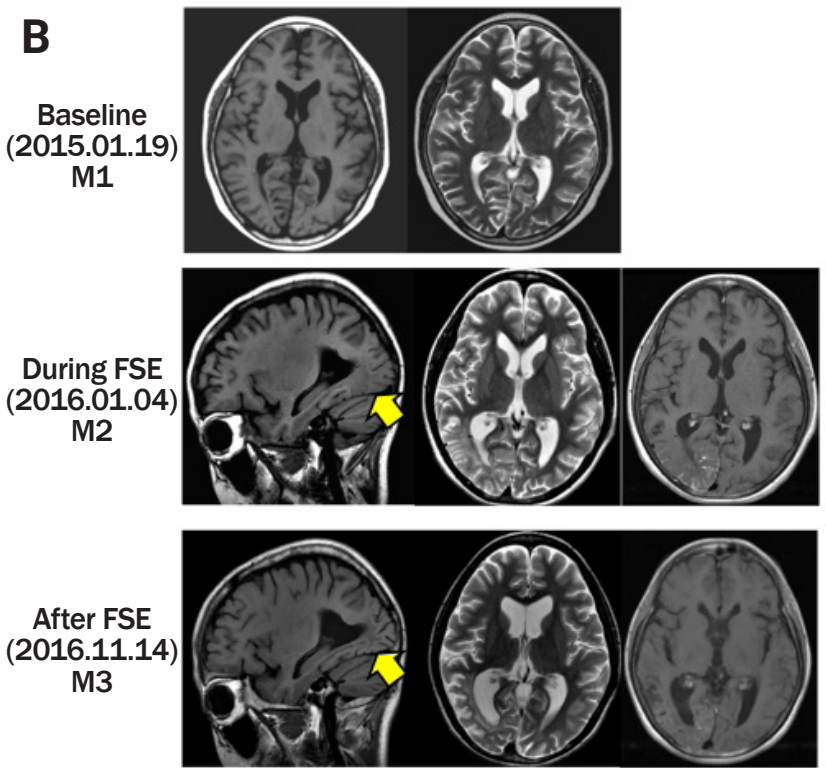

T1WI
T2WI
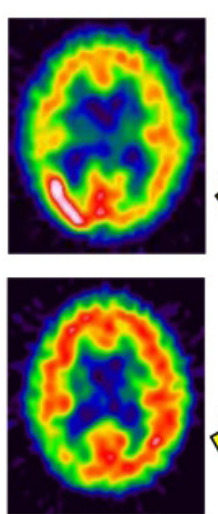

SPECT

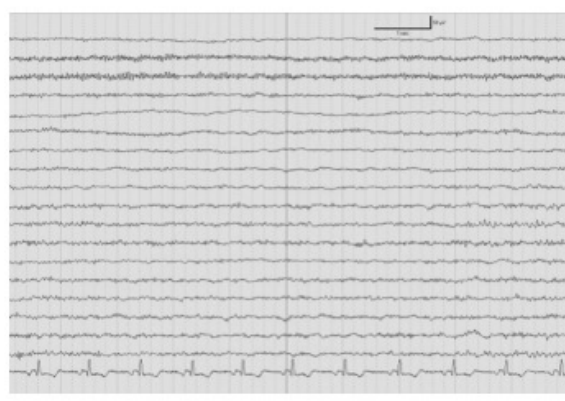

After FSE

(2016.04.20)
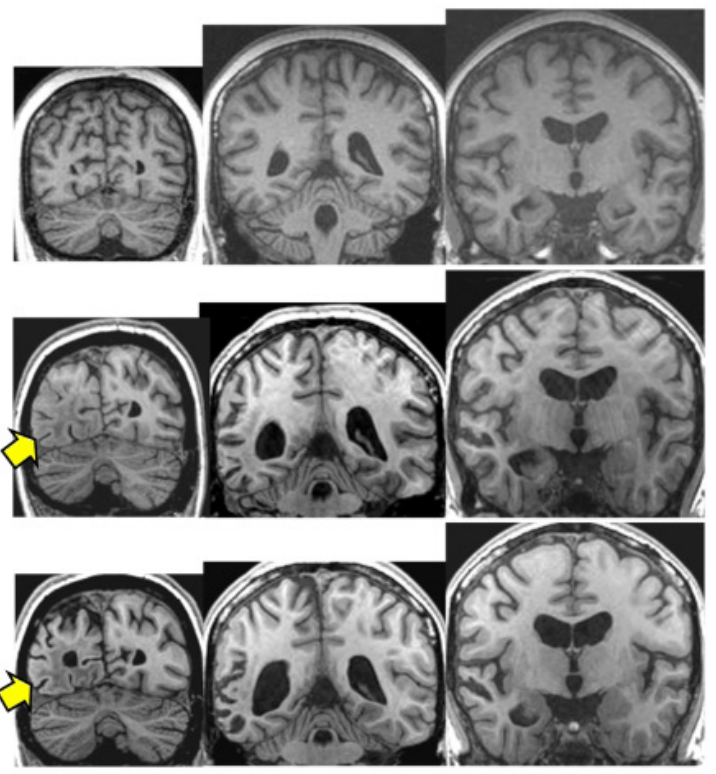

T1-volume MRI

(A) After proper treatment with high-dose clobazam (1 mg/kg/day), continuous ictal discharges over the right occipital area (left) resolved 1 week after admission (right). (B) Serial MRI. Images obtained at admission to the pediatric department (baseline, M1) showed no particular abnormalities except slight, diffuse cortical atrophy with mild ventricular dilatation of both sides. During and after incessant FSE, more prominent sulci and enlargement of the overlying subarachnoid spaces were observed mainly in the right occipital, temporoparietal, and hippocampal areas, in addition to ventricular dilatation (M2 and M3). Moreover, the site of prominent ictal hyperperfusion and leptomeningeal enhancement, as well as cortical laminar necrosis (yellow arrow) over the right occipitotemporal area, overlapped the area of high signal intensity of T2WI (M2). In follow-up MRI performed 10 months after cessation of FSE (M3), further atrophy was seen as ventricular dilatation and sulcal enlargement of subarachnoid space. Relative discrete hypoperfusion was observed in the same region on follow-up SPECT performed at 2 months after the first scan.

MRI, magnetic resonance imaging; SPECT, single-photon emission tomography; FSE, focal status epilepticus; T1WI, T1-weighted image; T2WI, T2-weighted image; GE-T1WI, gadolinium-enhanced T1WI. 
subsequent EEG displayed continuous paroxysmal fast activity in the right occipital area and frequent spike-and-waves over the right posterior temporooccipital region (not seen here). Before his second admission, regardless of the antiepileptic medication, he experienced focal onset impaired awareness seizures that manifested as oroalimentary automatism and unresponsiveness without any episodes of secondary generalization for more than 7 months.

In January 2016, he was readmitted to the neurology department of our hospital for management of uncontrolled partial SE. At the time of admission, his mental status was clear and oriented under medication of carbamazepine ( $800 \mathrm{mg} /$ day), topiramate (100 mg/day), and lamotrigine (100 mg/day). Serum level of carbamazepine was within the therapeutic range $(5.62 \mu \mathrm{g} / \mathrm{mL})$, while that of tacrolimus was low $(0.8 \mu \mathrm{g} / \mathrm{L})$. He showed normal blood pressure and no signs of infectious disease. Single-photon emission tomography (SPECT) and brain MRI (M2) performed that day revealed distinct hyperperfusion on the right temporooccipital area, which was matched to the lesion seen on MRI and the focal and perpetuating ictal discharges on scalp EEG (Figure 1A). During the tests, he incessantly experienced habitual visual hallucinations.

Fortunately, with an add-on of high-dose clobazam (1 mg/kg/ day), the fragmented, multicolored scintillation decreased markedly. A few days later, the symptom had completely resolved in conjunction with cessation of the EEG seizure pattern observed 5 days after clobazam add-on therapy (not seen here). The next week, he was discharged in a symptom-free state but complained of remnant left homonymous hemianopsia. Follow-up SPECT 2 months after the first scan showed discrete perfusion decrease in the corresponding areas (Figure 1B). In the EEG performed three months after cessation of visual symptoms, complete resolution of ictal epileptiform discharges and restoration to normal background (Figure 1A, 'after FSE') were found. Ten months after cessation of FSE, he was seizure-free and underwent follow-up MRI (M3) for identification of the interval changes in his brain.

\section{Single-photon emission tomography, magnetic resonance imaging acquisition, and volumetric analysis}

Two SPECT ('during FSE' and 'after FSE' in Figure 1B) images were acquired within 40 minutes after injection of $925 \mathrm{MBq}$ (25 mCi) ${ }^{99 \mathrm{~m}}$ Tc-ethyl cysteinate dimer. Using a dual-headed multidetector scanner (ECAM plus; Siemens, Erlangen, Ger- many) that was equipped with high-resolution low-energy fan-beam collimators and a full width at half maximum of 8.0 $\mathrm{mm}$, data were acquired as $128 \times 128$ matrices over a 40 -minute period during which each of the two heads made a $180^{\circ}$ orbit. Transaxial images were reconstructed using filtered back-projection. Attenuation correction was performed using Chang's algorithm with a $0.12 / \mathrm{cm}$ correction factor, and the calibration factor of $0.6 \mathrm{~mm} /$ pixel was applied for selection of the brain region.

MRI data from three stages (before FSE [M1], during FSE [M2], and post-FSE [10 months after cessation of FSE, M3]) (Figure 1B) were obtained with a 1.5-T Magnetom Vision Plus (Siemens; M1) and 3.0-T MRI machine (Skyra, Siemens; M2 and M3). The conventional routine examinations consisted of a standard fast spin-echo T2-weighted axial sequence (M1: TR/TE/NEX of 4,000/99/2, 6-mm thickness; M2 and M3: TR/ TE/NEX of 4,000/92/2, 5-mm thickness), axial fluid-attenuated inversion recovery (M1: 9,999/105/1; M2 and M3: 9,902/123/1), axial T1-weighted axial images (M1: 665/14/1; M2 and M3: 550/16/1) with gadolinium enhancement (only M2 and M3). In addition, three-dimensional T1-weighted coronal gradient-echo sequences (M1 [magnetization-prepared rapid gradient echo, MPRAGE]: 9.7/4.0/1; M2 and M3 [MPRAGE]: 22/6/1) were acquired in the coronal plane with $1.6 \mathrm{~mm}$ (M1) or $1.0 \mathrm{~mm}$ (M2 and M3) thickness for volumetric measurement.

The DICOM (Digital Imaging and Communications in Medicine) files of volumetric spoiled gradient echo (SPGR) images were transferred to a computer using a Windows operating system. Analyze 7.0 (Biomedical Imaging Resource, Mayo Foundation, 2006) was used for manual delineation of discrete anatomical structures. According to prior methods $[6,7]$, the volumes of cerebral structures were measured by one rater (Cho JH). To assess intra-rater reliability, each volume was measured three times, and the average value was selected to represent the volume status of three time points (M1, M2, and M3). The cerebral regional volume was computed by multiplying the surface of each region of interest by the slice thickness.

Conventional magnetic resonance imaging findings Contrary to the nonspecific findings, except for minimal cortical atrophy and slight ventricular dilation for his age, not only were prominent and widespread cortical atrophy manifested as salient sulci markings and markedly enlarged ventricles in M1and M2, but also remarkably high T2-signal intensities were recorded over the right occipitotemporal area (both gray 
and white matter were involved). Moreover, in the same cortical region, high signal intensity suggestive of cortical laminar necrosis (CLN) was observed in T1-weighted and SPGR images of M2, accompanied by the cortical gyral enhancement in the corresponding regions (yellow arrow in Figure 1B). Similar findings were observed in the follow-up images (M3), but there was some progression of parenchymal atrophy and higher intensity CLN at the right occipitotemporal area in which the FSE had occurred.

\section{Regional cerebral volume measured by magnetic resonance volumetry}

We performed consistent volumetric measurements, with intra-rater reliability coefficient of 0.975 (M1) to 0.994 (M2). Compared to M1, widespread regional atrophy was evident in M2, especially over the right posterior region (parietal, temporal, and occipital lobes) and right hippocampus. A remarkable decrease (greater than 5\%) of regional cerebral volume was determined not only in the ipsilateral hemisphere of ictal onset (occipital [17.6\%], parietal [8.7\%], and hippocampus [7.0\%]) but also in the contralateral side (left occipital [8.0\%], left parietal [5.0\%]). A significant volume change after cessation of FSE (regional volume decrease between M2 and M3) was evident only in right occipitotemporal areas (temporal, $13.8 \%$; occipital, $12.4 \%$ ), corresponding to the ictal onset zone of FSE (Figure 2).

\section{Discussion}

We described a patient under a relentlessly persisting FSE and subsequent irreversible neuronal damage as demonstrated by serial conventional MRI findings and corresponding MR volumetric measurement.

Our case report has some interesting points for discussion. First, unlike the changes demonstrated as T2 hyperintensity, leptomeningeal enhancement and cytotoxic or vasogenic edema, which are usually reversible, our case showed irreversible hyperattenuated T2 signal intensity after prolonged FSE. Similar to previous reports, early apparent diffusion coefficient (ADC) decrease from $14 \%$ to $49 \%$ of baseline value was maximal at 24 hours after seizure onset and normalized within several days. The ADC changes were correlated with the presumed area of seizure onset and the resulting histopathologic changes. The decrease of ADC in SE has been attributed to cytotoxic edema as a consequence of not ischemia but blood flow-metabolism uncoupling due to prolonged ictal discharge. Energy failure of the adenosine phosphatase pump and subsequent anaerobic glycolysis occur regardless of compensatory regional hyperperfusion.

Except for a few recent cases $[2,8]$, most studies have mentioned acute changes only during the short-term (a few hours to a few days) period of SE. Our patient, in contrast, showed T2 hyperintensity suggesting increased water content originating from increased extracellular space after tissue loss

Figure 2 Evolution of regional volume loss after FSE
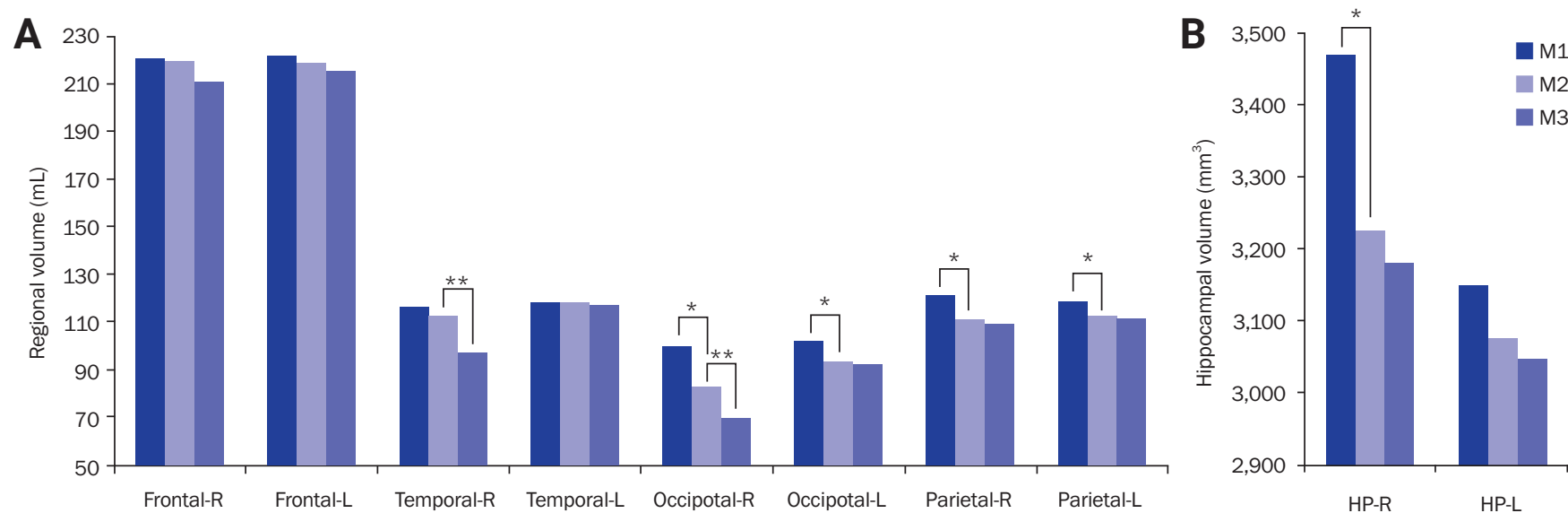

Compared to the first magnetic resonance imaging (before FSE), regional atrophy was discrete $(*)$, especially over the right posterior region (parietal, temporal, and occipital lobes) and right hippocampus (HP) identified with the volumetry data of M2 images. However, the volume changes after FSE (M3) were obvious in the right occipitotemporal areas $\left(^{* *}\right)$ but nowhere else (right frontal, parietal, and hippocampus and left hemisphere). FSE, focal status epilepticus; $R$, right; L, left. 
rather than from vascular or cytotoxic edema. Gadolinium enhancement was prominent in the ictal onset zone, where irreversible breakdown of the blood-brain barrier had persisted for a long time.

Second, with serial MR volumetry, we determined the transition of cerebral regional damage from prolonged FSE and identified the areas susceptible to intractable seizure-induced permanent atrophy. Progressive neuronal damage by SE has been confirmed by signs of ongoing neuronal cell death even after cessation of SE. A few case reports using MR changes over time have documented, despite full recovery of imaging abnormalities, some permanent brain injury such as enlargement of the lateral ventricle and sulci on the affected side, indicating volume loss after SE [3,5,8]. Moreover, studies have provided further evidence that hippocampal sclerosis can occur as a result of neuronal insult during SE $[4,8]$ and can develop within 2 months of SE and progress during the following 3 to 4 years.

In our case, repetitive cell firing from undertreated, prolonged FSE for at least several months resulted in marked regional atrophy manifested as widening of sulci and cortical thinning of areas adjacent or functionally connected to the ictal onset zone (M2). Moreover, progression of atrophy (right temporooccipital region, M3) was conspicuous in the region of discrete CLN.

Third, we assume that the CLN of our patient was primarily a consequence of FSE, considering its overlapping locus corresponding to the area displaying hyperperfusion shown by ictal SPECT as well as contemporary ictal EEG pattern. CLN is radiologically defined as high-signal cortical lesions on T1-weighted MRI images following a gyral distribution. Histopathologically, CLN is characterized by pannecrosis of the cortex involving neurons, glial cells, and blood vessels [9]. It has been reported to be associated with hypoxia, metabolic disturbances, drugs, and infections. Our patient showed focal neurological deficit (left hemianopia) after cessation of FSE that remained unchanged during follow-up. Therefore, prolonged FSE can cause permanent brain damage, as indicated by progression of concurrent CLN and its underlying regional cerebral atrophy in the absence of other metabolic or toxic causes.

Tacrolimus (FK506), one of the most popular immunosuppressants used in bone marrow and organ transplantation treatment, shows neurotoxicity by inhibiting phosphatase activity of calcineurin (CaN). The highly enriched distribution of
CaN in the dentate gyrus of the hippocampus suggests selective susceptibility of the hippocampus to injury from FK506. One case report showed MRI changes in hippocampal injury associated with tacrolimus [10], but the causal relationship between epileptic seizures and hippocampal damage from tacrolimus illustrated as high signal intensity of DWI in bilateral hippocampi has not been settled.

We cannot rule out that meningeal gadolinium enhancement was due to the effect of RPLS from tacrolimus or concomitant medical conditions. However, these are not likely the predominant cause of MRI changes considering several factors. First, our initial scan (M1) showed no cerebral edema involving a posterior lesion with hyperintensity in T2-weighted image. Second, the MRI features during and after FSE (M2, M3) were distinct from typical findings of RPLS in previous reports (usually bilateral, symmetrical, and sparing of calcarine fissure and paramedian occipital lobe areas). Third, during the period of relentless FSE, the patient did not experience any clinical or laboratory signs of infection or hypertension.

Our report has some limitations in that the serial MRI acquisition was performed with different MR machines (Siemens [M1] vs. GE Healthcare [M2 and M3]), with uneven acquisition parameters, and had discordant slice thickness of T1-volume images. However, as the volume decrease of the patient was more prominent than the predictable error from different MR machines measured for comparison, it seems justifiable to determine the pattern of interval changes of volume with our MR data.

In conclusion, we present a case in which a chronic focal SE caused not only prominent regional atrophy and its progression even after termination of the ictal process, but also concurrent CLN only on the same site, which was confirmed by conventional and volumetric MRI analyses.

\section{Conflicts of Interest}

No potential conflict of interest relevant to this article was reported.

\section{Author Contributions}

Conceptualization, Investigation: Lim SC, Shon Y; Methodology, Visualization: Cho JH; Supervision: Y Shon; Writing-original draft: Lim SC; Writing-review \& editing: Shon Y. 


\section{References}

1. Kim JA, Chung JI, Yoon PH, et al. Transient MR signal changes in patients with generalized tonicoclonic seizure or status epilepticus: periictal diffusion-weighted imaging. AJNR Am J Neuroradiol 2001;22:1149-1160.

2. Szabo K, Poepel A, Pohlmann-Eden B, et al. Diffusion-weighted and perfusion MRI demonstrates parenchymal changes in complex partial status epilepticus. Brain 2005;128(Pt 6):1369-1376.

3. Meierkord H, Wieshmann U, Niehaus L, Lehmann R. Structural consequences of status epilepticus demonstrated with serial magnetic resonance imaging. Acta Neurol Scand 1997;96:127-132.

4. Pohlmann-Eden B, Gass A, Peters CN, Wennberg R, Blumcke I. Evolution of MRI changes and development of bilateral hippocampal sclerosis during long lasting generalised status epilepticus. J Neurol Neurosurg Psychiatry 2004;75:898-900.

5. Lansberg MG, O'Brien MW, Norbash AM, Moseley ME, Morrell M, Albers GW. MRI abnormalities associated with partial status epilepticus. Neurology 1999;52:1021-1027.
6. Bokde AL, Teipel SJ, Schwarz R, et al. Reliable manual segmentation of the frontal, parietal, temporal, and occipital lobes on magnetic resonance images of healthy subjects. Brain Res Brain Res Protoc 2005; 14:135-145.

7. Pruessner JC, Li LM, Serles W, et al. Volumetry of hippocampus and amygdala with high-resolution MRI and three-dimensional analysis software: minimizing the discrepancies between laboratories. Cereb Cortex 2000;10:433-442.

8. Bauer G, Gotwald T, Dobesberger J, et al. Transient and permanent magnetic resonance imaging abnormalities after complex partial status epilepticus. Epilepsy Behav 2006;8:666-671.

9. Komiyama M, Nakajima H, Nishikawa M, Yasui T. Serial MR observation of cortical laminar necrosis caused by brain infarction. Neuroradiology 1998;40:771-777.

10. Yoshida Y, Shimada H, Mori T, Yoshihara H, Takeoka M, Takahashi T. FK506-associated limbic injury following umbilical cord blood transplantation. Bone Marrow Transplant 2003;32:523-525. 